(2) Open Access Full Text Article

\title{
Innovation of natural essential oil-loaded Orabase for local treatment of oral candidiasis
}

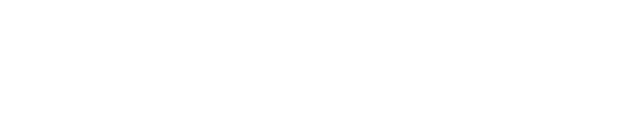

\author{
Gihan S Labib ${ }^{1,2}$ \\ Hibah Aldawsari' \\ 'Department of Pharmaceutics \\ and Industrial Pharmacy, Faculty of \\ Pharmacy, King Abdulaziz University, \\ Jeddah, Saudi Arabia; ' 2 Department of \\ Pharmaceutics, Faculty of Pharmacy, \\ Alexandria University, Alexandria, \\ Egypt
}

Purpose: Oral candidiasis may be manifested in the oral cavity as either mild or severe oral fungal infection. This infection results from the overgrowth of Candida species normally existing in the oral cavity in minute amounts based on many predisposing factors. Several aspects have spurred the search for new strategies in the treatment of oral candidiasis, among which are the limited numbers of new antifungal drugs developed in recent years. Previous studies have shown that thyme and clove oils have antimycotic activities and have suggested their incorporation into pharmaceutical preparations. This study aimed to investigate the possibility of the incorporation and characterization of essential oils or their extracted active ingredients in Orabase formulations.

Methods: Orabase loaded with clove oil, thyme oil, eugenol, and thymol were prepared and evaluated for their antifungal activities, $\mathrm{pH}$, viscosity, erosion and water uptake characteristics, mechanical properties, in vitro release behavior, and ex vivo mucoadhesion properties.

Results: All prepared bases showed considerable antifungal activity and acceptable physical characteristics. The release pattern from loaded bases was considerably slow for all oils and active ingredients. All bases showed appreciable adhesion in the in vitro and ex vivo studies.

Conclusion: The incorporation of essential oils in Orabase could help in future drug delivery design, with promising outcomes on patients' well-being.

Keywords: antifungal activity, clove oil, eugenol, mucoadhesion, oral gel, thyme oil, thymol

\section{Introduction}

Fungal oral infections have been increasing recently due to the growing number of high-risk patients. Such cases may be encountered in patients suffering from uncontrolled diabetes mellitus, patients with human immunodeficiency virus infection, patients with cancer or leukemia after receiving chemotherapy, patients who underwent transplantation surgeries, or those with prolonged use of broad-spectrum antibiotics. ${ }^{1-3}$

Oral candidiasis, which is sometimes referred to as oral thrush, may manifest as either mild or severe oral fungal infection resulting from the overgrowth of Candida species, which normally exists in the oral cavity in minute amounts. The incidence of severe cases of infection usually results from an impaired immune response. Recent studies in the US have revealed that Candida, among all fungal infectious species, represents the third or fourth most common isolate in nosocomial bloodstream samples. It is also considered the most common invasive fungal infection in critically ill nonneutropenic patients. ${ }^{4,5}$ Several aspects have spurred the search for new strategies in the treatment of oral candidiasis, among which are the limited numbers of new antifungal drugs developed in recent years, the increase of fungal resistance to traditional antifungal drugs, the high treatment costs, and the fungistatic activity of most of the available antifungal drugs..$^{6,7}$
Correspondence: Gihan S Labib Department of Pharmaceutics and Industrial Pharmacy, Faculty of Pharmacy, King Abdulaziz University, Building II, Jeddah 21589 , Saudi Arabia

Tel +96503027224

Email jilabib@gmail.com 
Several studies have also focused on discovering the possible inhibitory effect of many essential oils of plant origin, with their possible use as antimicrobial, antifungal, antiviral, antiparasitic, and insecticidal agents. ${ }^{8}$ Among the reported oils are cinnamon, clove, garlic, oregano, and thyme oil. These oils have been particularly selected and tested in the preservation of food. ${ }^{9}$

Many previous studies have shown that thyme (Thymus vulgaris) and clove (Syzygium aromaticum) have antimycotic activities and have suggested their incorporation into pharmaceutical preparations either as preservatives for cosmetic products or as drugs for the treatment of Candida infections. ${ }^{10-12}$ Other studies have specified that the main ingredients responsible for their antifungal activities are the phenolic compounds present mainly in thymol (thyme oil) and eugenol (clove oil). ${ }^{11,13}$ The strong antifungal activities of such essential oils could be of great utility in the treatment of oral thrush. It is worth mentioning that these oils have been used in folk medicine in different communities for patients suffering from oral candidiasis. ${ }^{14}$ However, their strong aromatic and corrosive action rendered them irritant and unsafe to be used in their crude form. ${ }^{15}$

Orabase is one of the most effective bioadhesive bases that have been used for pain relief in the management of oral aphthous stomatitis. It has been further used as a drug carrier containing $0.1 \%$ triamcinolone acetonide (Kenalog ${ }^{\circledR}$ ) for the same purpose. ${ }^{16,17}$ It is considered a hydrophobic gel, dental paste or sometimes is referred to as an ointment due to the presence of a high portion of liquid paraffin in its constituents.

The aim of this study was to investigate the possibility of incorporation of essential oils or their extracted active ingredients into Orabase for potential beneficial outcomes on patients' well-being.

\section{Materials}

Clove oil $(\mathrm{Cl})$ and thyme oil (TH) of commercial grade (El Kabten Company for aroma; Alexandria, Egypt), eugenol (Eug), thymol (Thy) $\geq 99.5 \%$, Folin-Ciocalteu (FC) reagent (Sigma-Aldrich Chemie GmbH, Buchs, Switzerland), carboxymethylcellulose sodium (CMC-Na) (Prolabo Pharmaceutical Chemicals Co, Cairo, Egypt), and gelatin (Gel) (ADWIC El-Nasr Pharmaceutical Chemicals Co, Cairo, Egypt) were used in the study. Pectin (Pect, industrial grade, with about $70 \%$ methyl esterification) was kindly supplied as a gift (Alamerya Pharmaceuticals, Alexandria, Egypt). Polyethylene resin was obtained from Kobosh Co (Alexandria,
Egypt). Other chemicals used were of pharmaceutical and analytical grades.

\section{Methods \\ Determination of minimum inhibitory concentration}

The minimum inhibitory concentration (MIC) was determined by applying the broth dilution method. ${ }^{11}$ Eugenol (Eug), clove oil $(\mathrm{Cl})$, and thyme oil $(\mathrm{TH}), 1 \mathrm{~mL}$ each, when weighed gave $1.010 \mathrm{~g}, 1.029 \mathrm{~g}$, and $1.001 \mathrm{~g}$, respectively. Dimethyl sulfoxide (DMSO), $500 \mu \mathrm{L}$, was added to a $500 \mu \mathrm{L}$ aliquot of each oil in small screw-capped glass bottles at room temperature and mixed well. Thymol crystals (Thy) were weighed and $0.5 \mathrm{~g}$ dissolved in $1 \mathrm{~mL}$ DMSO. After 15 minutes, doubling dilutions in duplicates, from 1:20 to 1:2,560, of all the prepared solutions were made in Sabouraud broth. An aliquot $(0.5 \mathrm{~mL})$ of Candida albicans (American Type Culture Collection 10231), at 105 colony-forming units $/ \mathrm{mL}(\mathrm{CFU} / \mathrm{mL})$, was added to each of the dilutions under study. A control sample of $0.5 \mathrm{~mL}$ of DMSO with $0.5 \mathrm{~mL}$ of C. albicans was prepared. The diluted samples and control were incubated at $37^{\circ} \mathrm{C}$ for 48 hours. The MIC of each sample under study was defined as the minimum concentration showing no visible growth.

\section{Preparation of placebo Orabase (oil-free Orabase)}

As stated in the Extra Pharmacopoeia, ${ }^{18}$ Orabase gel was prepared by mixing equal ratios of Orahesive base (CMCNa:Pect:Gel $=16.58 \%: 16.58 \%: 16.58 \%)$ and Plastibase (polyethylene [molecular weight: 21,000 ]: $5 \%$,w/w; liquid paraffin: $95 \%, w / w)$. The Orahesive base was prepared by first dispersing the calculated amount of Gel in hot water. After cooling, CMC-Na and Pect were added with continuous stirring. Plastibase was prepared by melting the required amount of polyethylene in liquid paraffin applying high temperature. A soft ointment base was formed after cooling. ${ }^{19}$

\section{Preparation of loaded Orabase}

The same procedure was adopted for the preparation of the oil-loaded base with the addition of the required amount of medicated oil or active ingredients in the final mixing step according to the base's loading capacity. Several trials were carried out to determine the maximum loading capacity of the base without showing any signs of physical instability or separation. The loading capacity of the base for $\mathrm{Cl}$, Eug, and TH oils was determined to be $40 \mathrm{mg} / \mathrm{g}$, and $8 \mathrm{mg} / \mathrm{g}$ for Thy in a finely divided powder form. 


\section{Determination of total phenolic content uniformity}

The total phenolic content was determined spectrophotometrically (double-beam ultraviolet-visible spectrophotometer; Pharmacia Biotech, Cambridge, England) using the FC method. ${ }^{20}$ This test is based on the oxidation of phenolic groups by phosphomolybdic and phosphotungstic acids (FC reagent). The products of the metal oxide reduction have a blue color that exhibits a broad light absorption with a maximum at $764 \mathrm{~nm}$ with an intensity that is proportional to the concentration of phenols. Twenty-microliter aliquot of the sample was added to $100 \mu \mathrm{L}$ of FC reagent. After 8 minutes, $300 \mu \mathrm{L}$ of saturated $25 \%$ sodium carbonate solution was added. The absorbance was measured at $764 \mathrm{~nm}$ after suitable dilution. The calibration curve was prepared using gallic acid solutions ranging from $0 \mathrm{mg} / \mathrm{L}$ to $500 \mathrm{mg} / \mathrm{L}$, and the results are given as gallic acid equivalents. This test was conducted in triplicate on all oils under study, their active ingredients, and the loaded Orabase preparations.

\section{Determination of $\mathrm{pH}$ of the prepared bases}

A suitable quantity $(1.5-\mathrm{g})$ of each base was accurately weighed and dispersed in $15 \mathrm{~mL}$ of purified water. The $\mathrm{pH}$ of the dispersions formed was measured using a $\mathrm{pH}$ meter (Systronics Digital DI-707, Ahmedabad, Gujarat, India).

\section{Effect of speed on the viscosity of Orabase formulations}

The viscosity of the prepared plain and medicated gel formulations was determined at $25^{\circ} \mathrm{C}$ using Brookfield viscometer (DV-II + Pro, Brookfield, Middleboro, MA, USA) with spindle number S-07. Measurements were made at speed settings ranging from $5 \mathrm{rpm}$ to $100 \mathrm{rpm}$, with a 30 -second interval between two successive speeds. The rheograms were constructed by plotting viscosity readings in centipoises (cP) versus spindle speed (rpm). ${ }^{21}$

\section{In vitro evaluation of the prepared base}

A membraneless dissolution model was adopted in the in vitro evaluation of the different Orabase formulations. The experiments were performed in a shaking water bath moving at 50 strokes $/ \mathrm{min}$ and maintained at $37^{\circ} \mathrm{C} \pm 0.5^{\circ} \mathrm{C}$. This model allows for the direct contact of the base with the dissolution medium (phosphate buffer [PB], $\mathrm{pH}=7.4$ ). ${ }^{22}$ Small wide-mouth vials containing $1 \mathrm{~g}$ of the base equally distributed at the bottom were placed in the water bath and
$1 \mathrm{~mL}$ of PB was layered over the base carefully to avoid mixing of the base upon addition of the release medium $(2 \mathrm{~mL})$. At predetermined time points, the entire release medium was removed for analysis, and the vials were weighed. Fresh previously warmed PB was layered carefully over the Orabase formulations. ${ }^{23}$ Each experiment was performed in triplicate.

\section{In vitro water uptake and erosion profiles} of Orabase formulations

After each removal of the release medium, the vials were carefully weighed and the cumulative percentage of water uptake or erosion of the bases was calculated in correspondence to the initial weight at zero time. Results were plotted versus time.

In vitro release of the oils and active ingredients from the prepared Orabase

The in vitro release of the oils or their active ingredients was analyzed simultaneously with the water uptake and erosion test. At the predetermined time point, the release medium was withdrawn completely and analyzed spectrophotometrically for the total phenolic content using the FC analysis method at a wavelength of $764 \mathrm{~nm} .{ }^{11,20,24}$ Release kinetics parameters, including zero-order, first-order, Higuchi, and KorsmeyerPeppas equations, were calculated for all release profiles to determine their release mechanism.

\section{Release profile comparisons}

Percentage dissolution efficiency (\%DE), mean dissolution time (MDT), and mean residence time (MRT) were calculated using the software package Microsoft Excel version 2007 and DD Solver, an add-in program for MS Excel for modeling and comparison of drug release profiles.

\section{Determination of the spreadability, shear stress, and adhesiveness}

The determination of spreadability and shear stress was done according to the previous studies with some modifications. ${ }^{25,26}$ Two smooth, polished glass blocks were selected; one block was fixed and leveled on a table. The upper block was passed down through a pulley by a thread, the end of which was tied to a pan. Then, $150 \mathrm{mg}$ of the freshly prepared base was weighed. Each separately was placed on the center of the fixed block. The second block was carefully placed and pressed with some weights, ranging from $50 \mathrm{~g}$ to $250 \mathrm{~g}$. The diameter formed by the gel between 
the blocks was measured after 5 minutes' application of each weight to indicate the spreadability of the gels. The same experiment was repeated using $200 \mathrm{~g}$, which represents the maximum spreadability for all bases under study. After 5 minutes of application, weights were added to the attached pan. The weights required to pull the block or to make it slide down from the base block represented shear stress, adhesion strength, in grams.

The force required to overcome the attraction force between the surface and the sample (adhesiveness) was measured by the plate agar method. Agar plates (2\% agar, $\mathrm{w} / \mathrm{v}$, in $\mathrm{PB}$ [pH 7.4]) of $5 \mathrm{~cm}$ diameter were prepared. A small quantity $(0.5 \mathrm{~g})$ of the base was placed on the center of the agar plate, making a circle of $5 \mathrm{~mm}$ in diameter. The plate was slanted at $30^{\circ}$ for 3 hours, and the distances moved by the samples were measured at room temperature at predetermined time periods. ${ }^{27}$

\section{Ex vivo mucoadhesion study}

Albino rabbits weighing $2.25 \mathrm{~kg}$ to $2.5 \mathrm{~kg}$ were sacrificed immediately before the experiment, and the buccal mucosa was removed and separated from all surrounding tissues and muscles according to a previously reported method. ${ }^{28}$ After rinsing, the tissues were fixed to the glass slides by means of glue. The ex vivo mucoadhesion study was performed by the application of Orabase on the fresh-glued mucosa. The mucoadhesive base was wetted with 1 drop of PB (pH 7.4) and pasted to the mucosa by applying a light force with a stainless steel spatula for 30 seconds. The glass slide was introduced into a beaker filled with $100 \mathrm{~mL}$ of PB, pH 7.4, and maintained in a shaking water bath at $37^{\circ} \mathrm{C} \pm 0.5^{\circ} \mathrm{C}$, 50 strokes/min. The Orabase adhesion was monitored for 6 hours. The time for complete washing out of the base layer from the mucosa was determined. ${ }^{29}$

\section{Results}

\section{Determination of MIC}

Comparison between the antifungal activities of the commercial essential oils and their active phenolic ingredients is shown in Table 1. Cl and TH oils had mean MIC values of $3.2 \times 10^{3} \mu \mathrm{g} / \mathrm{mL}$ and $0.78 \times 10^{3} \mu \mathrm{g} / \mathrm{mL}$, respectively, while Eug and Thy had values of $3.1 \times 10^{3} \mu \mathrm{g} / \mathrm{mL}$ and $0.79 \times 10^{3} \mu \mathrm{g} / \mathrm{mL}$, respectively. The control sample containing DMSO showed no inhibition effect on Candida species at the concentration used.

\section{Preparation of placebo and loaded Orabase}

All prepared Orabase formulations showed good pasty consistency. However, Orabase loaded with essential oils and Eug had a more shiny texture compared to Orabase loaded with Thy fine powder and the nonloaded one.

\section{Determination of total phenolic content and content uniformity}

Results are presented in Table 2. It could be seen that the phenolic contents in the crude ingredients were comparable to those obtained from the formulations. Percentage recovery after dissolution was also calculated to ensure homogeneous distribution of the active ingredients in the base.

Table I Minimum inhibitory concentrations of active ingredients used in Orabase

\begin{tabular}{|c|c|c|c|c|c|c|c|c|}
\hline \multirow{2}{*}{$\begin{array}{l}\text { Candida albicans } \\
\text { (ATCC I023I) }\end{array}$} & \multicolumn{8}{|c|}{ Dilutions } \\
\hline & $1 / 20$ & $1 / 40$ & $1 / 80$ & $1 / 160$ & $1 / 320$ & $1 / 640$ & $1 / 1,280$ & $1 / 2,560$ \\
\hline \multirow[t]{3}{*}{ Clove oil } & - & - & - & - & - & + & + & + \\
\hline & - & - & - & - & + & + & + & + \\
\hline & - & - & - & - & + & + & + & + \\
\hline Concentration $(\mu \mathrm{g} / \mathrm{mL})$ & $2.5 \times 10^{4}$ & $1.3 \times 10^{4}$ & $6.4 \times 10^{3}$ & $3.2 \times 10^{3}$ & $1.6 \times 10^{3}$ & $8 \times 10^{2}$ & $4 \times 10^{2}$ & $2 \times 10^{2}$ \\
\hline \multirow[t]{3}{*}{ Eugenol } & - & - & - & - & + & + & + & + \\
\hline & - & - & - & - & + & + & + & + \\
\hline & - & - & - & - & - & + & + & + \\
\hline Concentration $(\mu \mathrm{g} / \mathrm{mL})$ & $2.5 \times 10^{4}$ & $1.2 \times 10^{4}$ & $6.3 \times 10^{3}$ & $3.1 \times 10^{3}$ & $1.6 \times 10^{3}$ & $7.9 \times 10^{2}$ & $3.9 \times 10^{2}$ & $1.9 \times 10^{2}$ \\
\hline \multirow[t]{3}{*}{ Thyme oil } & - & - & - & - & - & - & - & + \\
\hline & - & - & - & - & - & - & + & + \\
\hline & - & - & - & - & - & - & + & + \\
\hline Concentration $(\mu \mathrm{g} / \mathrm{mL})$ & $2.5 \times 10^{4}$ & $1.2 \times 10^{4}$ & $6.2 \times 10^{3}$ & $3.2 \times 10^{3}$ & $1.6 \times 10^{3}$ & $7.8 \times 10^{2}$ & $3.9 \times 10^{2}$ & $1.9 \times 10^{2}$ \\
\hline \multirow[t]{3}{*}{ Thymol } & - & - & - & - & - & - & + & + \\
\hline & - & - & - & - & - & - & - & + \\
\hline & - & - & - & - & - & - & + & + \\
\hline Concentration $(\mu \mathrm{g} / \mathrm{mL})$ & $2.5 \times 10^{4}$ & $1.2 \times 10^{4}$ & $6.3 \times 10^{3}$ & $3.1 \times 10^{3}$ & $1.6 \times 10^{3}$ & $7.9 \times 10^{2}$ & $3.9 \times 10^{2}$ & $1.9 \times 10^{2}$ \\
\hline
\end{tabular}

Notes: Each test was done in triplicate. The bold numbers represent the determined minimum inhibitory concentration. Abbreviations: -, no growth; +, growth of microorganism. 
Table 2 Total phenolic content of essential oils, total content uniformity, and percentage recovery of phenolic content from the prepared Orabase formulations

\begin{tabular}{|c|c|c|c|c|}
\hline & $\mathrm{Cl}$ & Eug & TH & Thy \\
\hline $\begin{array}{l}\text { Total phenolic content in } 20 \mu \mathrm{L} \\
\text { crude sample } \mu \mathrm{g} / \mathrm{mL}( \pm \text { SD) }\end{array}$ & $88.70 \times 10^{2}( \pm 24.7)$ & $118.77 \times 10^{2}( \pm 42)$ & $122.7 \times 10^{2}( \pm 9.08)$ & $86.98 \times 10^{2}( \pm 54.5)^{a}$ \\
\hline $\begin{array}{l}\text { Total phenolic content uniformity } \\
\text { in I } g \text { base, } \mu g / m L( \pm S D)\end{array}$ & $177.25 \times 10^{2}( \pm 49.4)$ & $237.33 \times 10^{2}( \pm 84)$ & $245.4 \times 10^{2}( \pm 18.16)$ & $27.81 \times 10^{2}( \pm 17.5)$ \\
\hline $\begin{array}{l}\% \text { total phenolic content recovery } \\
\text { from bases after release experiment }\end{array}$ & $101.34 \pm 0.48$ & $103.54 \pm 0.54$ & $101.6 \pm 1.009$ & $102.72 \pm 0.67$ \\
\hline
\end{tabular}

Note: a Amount in $25 \mathrm{mg}$ sample of thymol crystals.

Abbreviations: $\mathrm{Cl}$, clove oil; Eug, eugenol; SD, standard deviation; $\mathrm{TH}$, thyme oil; Thy, thymol.

\section{Determination of $\mathrm{pH}$ of the prepared bases}

All bases under study showed a nearly neutral $\mathrm{pH}$ ranging from 6.8 to 7.1, as seen in Table 3 .

\section{Effect of speed on the viscosity of Orabase formulations}

A general pattern was observed for all prepared bases, including the nonloaded one, whereby an increase in the angular velocity caused a remarkable decrease in the measured viscosity with a nearly straight-line pattern at higher speeds. It is worth stating that all loaded bases showed a decrease in viscosity compared to the nonloaded base (Figure 1).

\section{In vitro evaluation of the prepared base In vitro water uptake and erosion profiles of Orabase formulations}

The water uptake and erosion profiles are presented in Figure 2. It could be observed that the nonloaded base showed erosion resistance properties when compared to the loaded ones. $\mathrm{Cl}$ Orabase showed slow water uptake for the 1 st hour, after which erosion started at a very slow rate, reaching $12 \%$ cumulative erosion by the end of the study. This effect was less pronounced with Eug-loaded Orabase, whereby the base showed slow water uptake for the first half hour, followed by a higher erosion rate, reaching

Table 3 Values of $\mathrm{pH}$ and release kinetics of prepared Orabase formulations

\begin{tabular}{llllll}
\hline Orabase & $\mathbf{p H} \pm \mathbf{S D}$ & $\begin{array}{l}\text { Zero } \\
\text { order }\end{array}$ & $\begin{array}{l}\text { First } \\
\text { order }\end{array}$ & Higuchi & $\begin{array}{l}\text { Korsmeyer- } \\
\text { Peppas }\end{array}$ \\
\hline $\mathrm{Cl}$ & $7.1 \pm 0.05$ & 0.9227 & 0.9653 & 0.9857 & 0.472 \\
Eug & $6.9 \pm 0.25$ & 0.9742 & 0.9961 & 0.9920 & 0.692 \\
$\mathrm{TH}$ & $7.1 \pm 0.15$ & $0.978 \mathrm{I}$ & 0.9969 & 0.9945 & 0.639 \\
Thy & $6.8 \pm 0.2$ & 0.9716 & 0.9882 & 0.9685 & 0.795 \\
$\mathrm{PL}$ & $7.1 \pm 0.05$ & - & - & - & - \\
\hline
\end{tabular}

Abbreviations: $\mathrm{Cl}$, clove oil; Eug, eugenol; PL, placebo; SD, standard deviation; TH, thyme oil; Thy, thymol. about $65 \%$ cumulative erosion by the end of the study. As for TH oil- and Thy-loaded bases, they had nearly the same behavioral patterns of erosion from the start of the experiment to the end, with lower extent in the case of the latter, reaching $98 \%$ and $80 \%$ cumulative erosion, respectively.

In vitro release of the oils and active ingredients from the prepared Orabase

The in vitro release of $\mathrm{Cl}$ from Orabase was more pronounced initially for the first hour compared to the release of Eug. In the case of TH oil, there was a general increase in the release rate compared to Thy release profile, reaching about $90 \%$ by the end of the study. After the 3rd hour of release, $\mathrm{Cl}$, Eug, and Thy release profiles were comparable to each other until the end of experiment, whereby nearly all of them released $60 \%$ of their content (Figure 3 ). The release kinetics calculated for all formulations showed a mixed mechanism of release in most cases (Table 3).

Comparison of the release profiles by calculating $\% \mathrm{DE}$, MDT, and MRT revealed a slight difference among the tested bases in the controlled release pattern (Table 4).

\section{Determination of the spreadability, shear stress, and adhesiveness}

Moderate increase in spreadability was observed for all prepared bases under study (Figure 4). Both the shear stress required to move the plate and the adhesiveness was in the following order (Figures 5 and 6): Placebo $>\mathrm{Eug}>\mathrm{Cl}>$ TH $>$ Thy.

\section{Ex vivo mucoadhesion study}

All Orabase formulations under study showed strong mucoadhesion to the model mucosa for $>6$ hours.

\section{Discussion}

The antibacterial and antifungal activities possessed by essential oils was suggested by several researchers to be useful in 

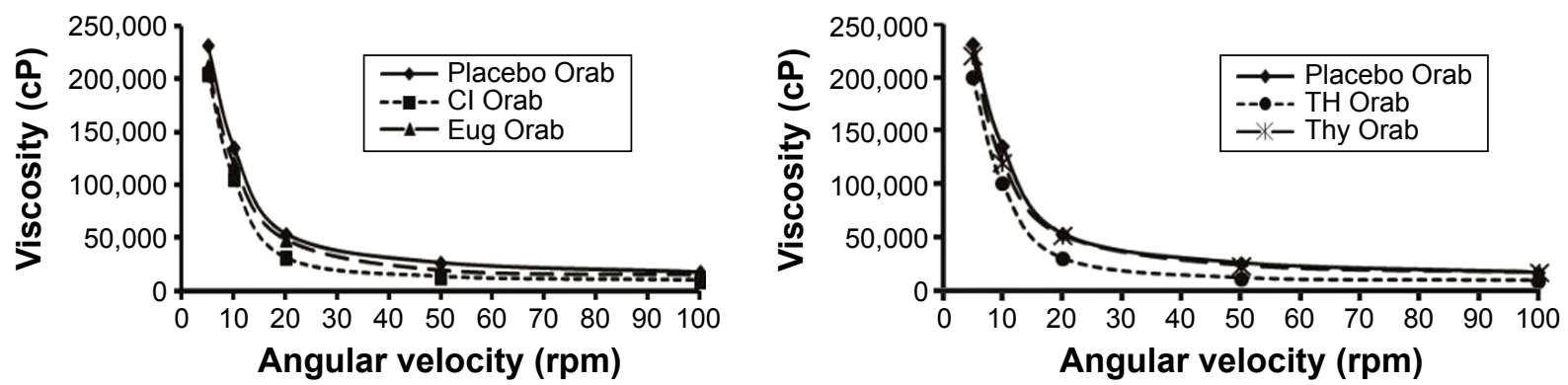

Figure I Rheograms of the prepared loaded Orabase formulations in comparison to nonloaded Orabase. Abbreviations: Cl, clove oil; Eug, eugenol; Orab, Orabase; TH, thyme oil; Thy, thymol.

pharmaceutical preparations, with special focus on the use of $\mathrm{Cl}$ and $\mathrm{TH}$ oils in the treatment of candidiasis. ${ }^{30-32}$

The MIC values of the commercial oils and their main active ingredients were determined to confirm their antifungal activity against the tested organism. All studied samples showed remarkable inhibitory effect on C. albicans. The maximum inhibitory effect was observed with $\mathrm{TH}$ oil and Thy crystals, compared to the effect of $\mathrm{Cl}$ and Eug. It is worth mentioning, however, that the constituents of the natural oils differ in their yield, concentration, and amount recovered based on several factors, including species of plant, method of extraction, solvents used, and extraction time, which in turn may differ in their antifungal potency. ${ }^{33,34}$ Although some studies showed different MIC results, ${ }^{31}$ results comparable to our determinations were observed in a previously reported study. ${ }^{11}$

\section{Physical characteristics}

Several preliminary trials were made to determine the maximum loading capacity of the Orabase prepared with the oils and their active ingredients. All oils and active ingredients used are lipophilic in nature, which rendered the possibility of high loading capacity in the solid lipophilic base used with maximum stability. However, if the amount of liquid lipid exceeds its solubility in the solid lipophilic base, phase separation is liable to occur. ${ }^{35}$ The maximum loading capacity without any sign of base instability was determined and it represented about $4 \%$ for both oils and Eug $(40 \mathrm{mg} / \mathrm{g})$ and $0.8 \%$ for Thy $(8 \mathrm{mg} / \mathrm{g})$ in a finely divided form. Although there is no recommended dose for such oils, there are some reported works on their irritant and toxic amounts, far greater than the doses used in our study. ${ }^{31,36,37}$ However, further preclinical studies are needed for confirmation of their safety in the doses used. The neutral $\mathrm{pH}$ of the prepared Orabase will probably ensure its applicability and biocompatibility in the oral cavity with minimal possible irritation. ${ }^{38}$ Moreover, the general increase in viscosity of the mucoadhesive prepared Orabase will help in its bioretention in the oral cavity. ${ }^{39}$ This increase in viscosity could be attributed to the presence of high concentrations of different hydrophilic polymers embedded in the lipophilic base. ${ }^{40}$ However, the incorporation of the lipophilic active ingredients contributed to the slight decrease of the viscosity, as observed in the results. This can be attributed to the possible decrease of cohesiveness of the hydrophilic polymers to each other and the possible decrease of the H-bonding between them. ${ }^{41}$ The shape of the
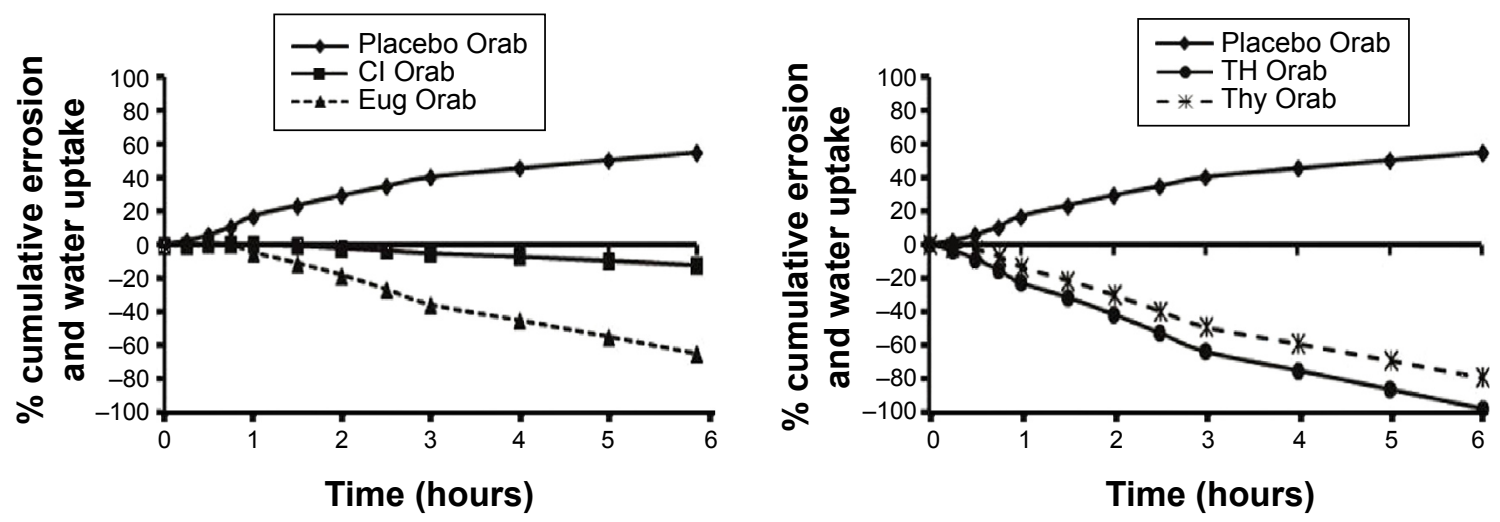

Figure 2 Percentage cumulative erosion and water uptake by the prepared loaded Orabase formulations in comparison to the nonloaded base. Abbreviations: $\mathrm{Cl}$, clove oil; Eug, eugenol; Orab, Orabase; TH, thyme oil; Thy, thymol. 

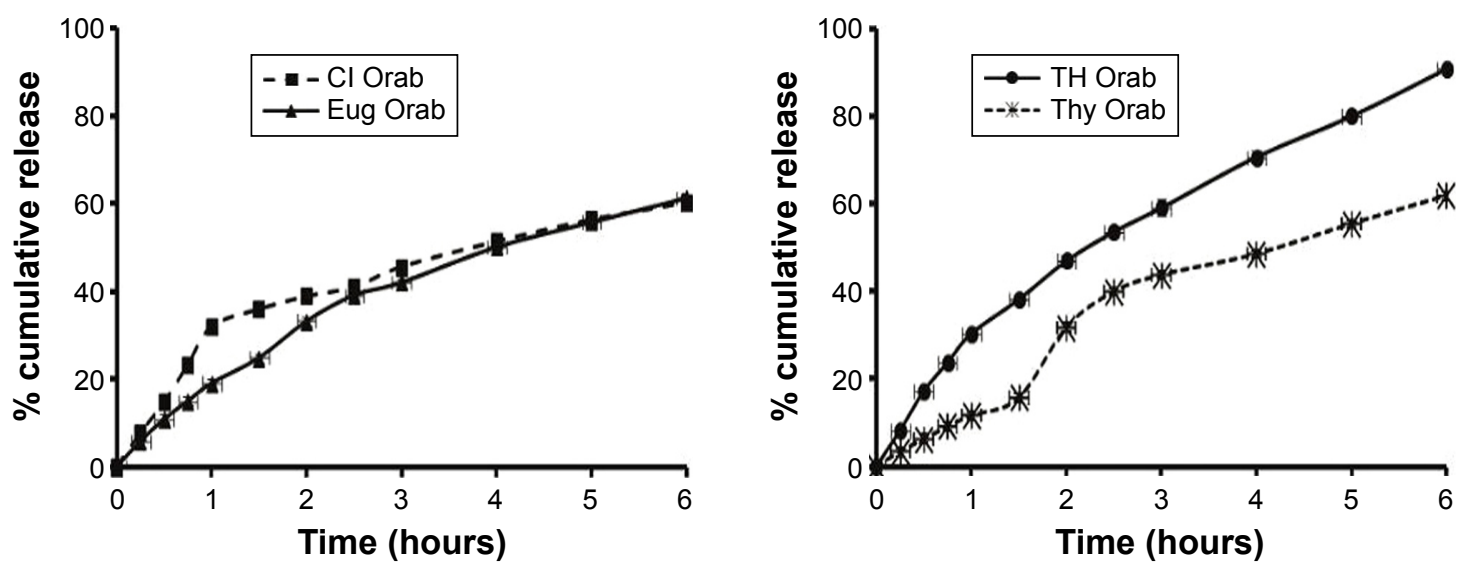

Figure 3 Percentage cumulative release profiles of the active ingredients from the prepared loaded Orabase formulations. Abbreviations: $\mathrm{Cl}$, clove oil; Eug, eugenol; Orab, Orabase; TH, thyme oil; Thy, thymol.

rheograms showed shear thinning behavior (pseudoplastic), wherein at lower angular velocity, the rate of decrease in the viscosity is much higher than that at higher one. At higher speeds, the curves tend to be straight. This phenomenon was previously explained in several studies on the bases of the presence of the solvent within the entangled molecules in the three-dimensional network structure of the base at lower shear rate (speed). Upon increasing the shear rate, the molecule became disentangled and showed less resistance to flow, with the release of some of the entrapped water in the destroyed network structure. This character is required in topical pharmaceutical bases. ${ }^{42,43}$

\section{Determination of total phenolic content and content uniformity}

Several studies reported the presence of Eug and Thy as the main components of clove oil and thyme oil, respectively. ${ }^{15,37}$ Being of phenolic origin, they could be detected in pharmaceutical preparations using the FC spectrophotometric method. Results obtained applying this method were reproducible and accurate, as indicated by the average percentage recovery ranging from $101 \%$ to $103 \%$ from all loaded Orabase preparations. It is worth indicating that Thy Content was also determined in mouthwashes in a previous

Table 4 Comparative parameters of the release profiles of loaded Orabase formulations after 6 hours

\begin{tabular}{lllll}
\hline Parameter & Cl & Eug & TH & Thy \\
& Orabase & Orabase & Orabase & $\begin{array}{l}\text { Orabase } \\
\text { DE (\%) }\end{array}$ \\
MRT (hours) & 2.58 & 38.6 & 42.3 & 36.7 \\
MDT (hours) & 1.79 & 2.52 & 2.45 & 2.49 \\
\hline
\end{tabular}

Abbreviations: $\mathrm{Cl}$, clove oil; $\mathrm{DE}$, dissolution efficiency; MDT, mean dissolution time; MRT, mean residence time; Eug, eugenol; TH, thyme oil; Thy, thymol. study using a similar spectrophotometric method with great success. ${ }^{44}$

\section{In vitro evaluation of the prepared base In vitro water uptake and erosion profiles of Orabase formulations}

The nonloaded base showed an increase in water uptake and resistance to erosion from the start to the end of the study, probably due to the high content of swellable polymers embedded in the lipophilic base, which enhances water uptake rather than erosion. This fact decreased markedly by loading the base with either the oils or their active ingredients probably due to the disruption of the hydrophilic molecular associations and thus loosening the structured rigid base, allowing erosion to happen rather than water uptake. However, this effect was more pronounced with TH and Thy, while $\mathrm{Cl}$ and Eug showed more resistance to erosion. This could be explained by the differences in the nature of the oils and active ingredients used, with a possible difference in their interactions with the Orabase ingredients.

In vitro release of the oils and active ingredients from the prepared Orabase

The in vitro release method simulated the in vivo condition in allowing the regular washout of the medium and its renewal, resembling the salivary flow in the buccal cavity. Moreover, the membraneless model for drug release from oral gels and bases would exclude any possible factors while allowing direct contact of the base with the medium. ${ }^{22}$ Sink condition was also maintained by the total replacement of the media at predetermined times. Release profiles indicated a slow controlled release rate with respect to all added oils and active ingredients. However, $\mathrm{Cl}$, Eug, and TH Orabase 


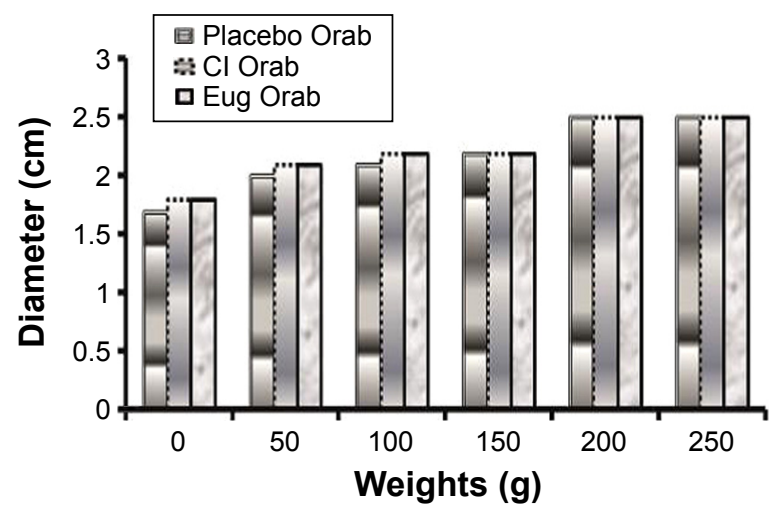

Figure 4 Spreadability study on the nonloaded and loaded Orabase formulations. Abbreviations: $\mathrm{Cl}$, clove oil; Eug, eugenol; Orab, Orabase; TH, thyme oil; Thy, thymol.

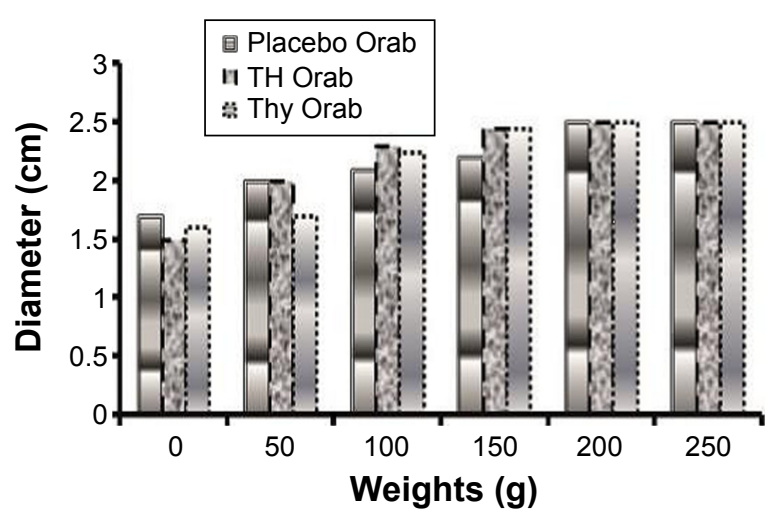

preparations showed a biphasic release pattern, starting with a slight burst effect releasing about $31 \%, 30 \%$, and $20 \%$ in the 1 st hour, respectively. This was followed by a slow release profile until the end of the study in the case of $\mathrm{Cl}$ and Eug, while a higher release pattern was observed for TH oil. A similar study showed the same release pattern from lipophilic nanoparticles loaded with Eug. ${ }^{35}$ This phenomenon was explained based on the concept of the possible association of the lipophilic drug with the lipophilic base depending on its melting point. ${ }^{45}$ On the other hand, this could be further explained based on the possible initial release of active ingredients from the surface layer of the base. For Thy-loaded Orabase, the initial delay in the release pattern could be explained by the fact that Thy in the solid state would be embedded in the lipophilic matrix, resulting in a slower release rate. The general prolonged release rate from all prepared Orabase formulations could be attributed to the high viscosity of the used base, with a consequent decrease in the penetration rate of fluid. ${ }^{26,46}$ Drug release from bases in general may follow different mechanisms. In our study, it was observed that the drug release is governed by both diffusion and dissolution of the active ingredients (Table 4). The values of $R^{2}$ in the cases of $\mathrm{Cl}$, Eug, and TH were found to be high for the Higuchi model, indicating that the main mechanism of their release from the base is by diffusion. This is in accordance with results of a previous study on the release of eugenol from a lipid nanostructured formulation. ${ }^{35}$ Thy release was governed mainly by dissolution. However, for all loaded bases, the dissolution of drug from the eroded base had an important role, as indicated by the high $R^{2}$ values (first order). These results were in accordance with those of a previous study on the release kinetics from other bases. ${ }^{21}$

\section{Determination of the spreadability, shear stress, and adhesiveness}

Spreadability and adhesiveness serve in determining the clinical applicability and patient compliance of the prepared base. ${ }^{26,47}$ The increase in polymer concentration within the

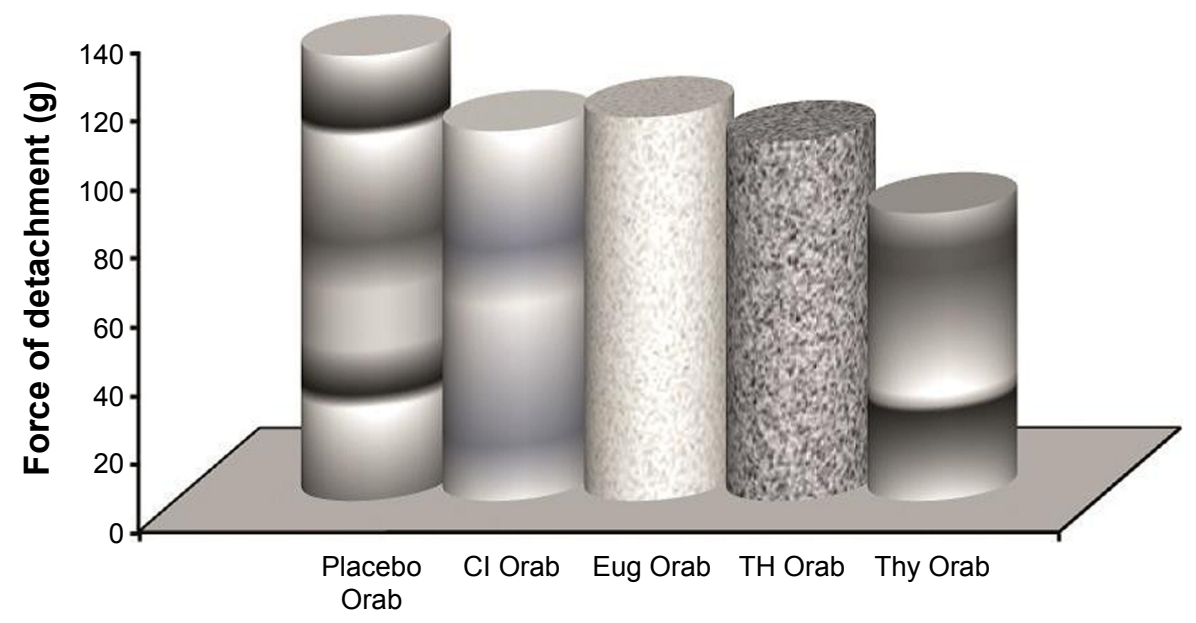

Figure 5 Shear stress study on the nonloaded and loaded Orabase formulations.

Abbreviations: $\mathrm{Cl}$, clove oil; Eug, eugenol; Orab, Orabase; TH, thyme oil; Thy, thymol. 

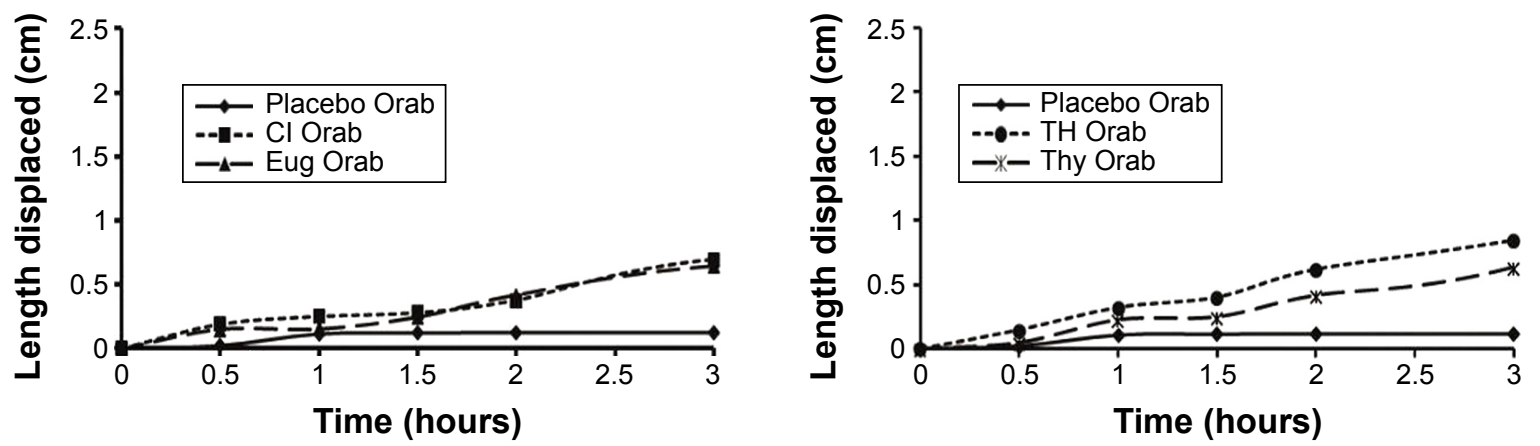

Figure 6 Comparative agar-adhesiveness study on the nonloaded and loaded Orabase formulations. Abbreviations: $\mathrm{Cl}$, clove oil; Eug, eugenol; Orab, Orabase; TH, thyme oil; Thy, thymol.

base may have contributed to the moderate values of spreadability for all prepared bases. ${ }^{47}$ However, it was observed that applying more weights had little effect on the increase of spreadability. This indicates that the minimum application of stress would produce the maximum spreading effect on the base prepared. On the other hand, the force required to displace the plate showed satisfactory adhesion property of all loaded bases. These results were confirmed with the small displacement length values on agar plates. Both tests indicated appropriate adhesiveness that could reflect strong in vivo mucoadhesive properties of the prepared bases.

\section{Ex vivo mucoadhesion study}

Mucoadhesion serves as an important parameter for the in vivo retention of the formulation in the oral cavity, which in turn would increase and prolong its efficacy. It was previously reported that the increase in concentration of polymers in the base plays an effective role in the increased mucoadhesion strength. The high-molecularweight swellable polymers would allow for entire contact with the mucin and for better adhesion. This was observed in our study by the increase in the ex vivo retention time of all formulations over a period of 6 hours. ${ }^{48}$ This observed retention phenomenon was confirmed by clinical trials on patients surfing from oral thrush using the prepared Orabase formulations. The effectiveness of the enhanced treatment of such cases was also confirmed with a remarkable decrease in microbial count. ${ }^{49}$

\section{Conclusion}

Clove oil, thyme oil, and their main active ingredients present in eugenol and thymol were evaluated for their antifungal activity against $C$. albicans. Orabase formulations loaded with oils and their active ingredients were prepared and evaluated for their physical characteristics, total phenolic content, viscosity, in vitro erosion and water uptake properties, and in vitro release. They were also evaluated for their spreadability, adhesiveness, and ex vivo mucoadhesion properties. All loaded bases showed appropriate physical properties with no sign of separation. Erosion of bases was limited to some extent with respect to clove oil and eugenol while being more pronounced with respect to thyme oil and thymol. A controlled release pattern of the active ingredients was observed and this was governed by mixed mechanisms of diffusion and dissolution release kinetics. Although the spreadability was somehow moderate, the in vitro and ex vivo adhesiveness showed promising results for the applicability of Orabase in natural settings.

It is suggested that further studies on different topical bases be conducted for the loading of natural oils and active ingredients to decrease the risk that may accompany the use of such ingredients in folk medicine in their crude form.

\section{Acknowledgments}

This work was supported by the Deanship of Scientific Research (DSR), King Abdulaziz University, Jeddah, under grant number 166-666-D1435. The authors, therefore gratefully acknowledge the DSR technical and financial support.

The authors also sincerely thank Professor Mahmoud Yassin and Dr Mahmoud Abdelkhalik, Department of Microbiology, Faculty of Pharmacy, King Abdulaziz University, for their help in the determination of the minimum inhibitory concentrations. The authors would like to declare that our submitted paper "Innovation of natural essential oil-loaded Orabase for local treatment of oral candidiasis" was presented in a poster session at the American Association of Pharmaceutical Scientists (AAPS) Annual Meeting and Exposition held at San Diego in 2014, with no detailed information on the work done. The poster's abstract was published in "Poster Abstracts" in the AAPS conference book (https://2014aapsam.zerista. com/poster/member/23075). The actual paper, however, has not been published in any journal. 


\section{Disclosure}

The authors report no conflicts of interest in this work.

\section{References}

1. Terrell C, Hughes CE. Antifungal agents used for deep-seated mycotic infections. Mayo Clin Proc. 1992;67:69-91.

2. Keele DJ, DeLallo VC, Lewis RE, Ernst EJ, Klepser ME. Evaluation of amphotericin B and flucytosine in combination against Candida albicans and Cryptococcus neoformans using time-kill methodology. Diag Microbiol Infect Dis. 2001;4:121-126.

3. Pappas PG, Rex JH, Lee J, et al; NIAID Mycoses Study Group. A prospective observational study of candidemia: epidemiology, therapy, and influences on mortality in hospitalized adult and pediatric patients. Clin Infect Dis. 2003;37:634-643.

4. Wisplinghoff $\mathrm{H}$, Bischoff $\mathrm{T}$, Tallent SM, Seifert H, Wenzel RP, Edmond MB. Nosocomial bloodstream infections in US hospitals: analysis of 24,179 cases from a prospective nationwide surveillance study. Clin Infect Dis. 2004;39:309-317.

5. Marchetti O, Bille J, Fluckiger U, et al; Fungal Infection Network of Switzerland. Epidemiology of candidemia in Swiss tertiary care hospitals: secular trends, 1991-2000. Clin Infect Dis. 2004;38:311-320.

6. Rapp RP. Changing strategies for the management of invasive fungal infections. Pharmacotherapy. 2004;24:4S-28S.

7. Kauffman CA. Fungal infections. Proc Am Thorac Soc. 2006;3:35-40.

8. Bakkali F, Averbeck S, Averbeck D, Idaomar M. Biological effects of essential oils - a review. Food Chem Toxicol. 2008;46:446-475.

9. Conner D, Beuchat L. Sensitivity of heat-stressed yeast to essential oils of plants. Appl Environ Microbiol. 1984;47:229-233.

10. Manou I, Bouillard L, Devleeschouwer MJ, Barel AO. Evaluation of the preservative of Thymus vulgaris essential oil in topically applied formulations under a challenge test. J Appl Microbiol. 1998;84: $368-376$.

11. Nzeako B, Al-Bushra L. Comparative studies of antimycotic potential of thyme and clove oil extracts with antifungal antibiotics on Candida albicans. Afr J Biotechnol. 2008;7(11):1612-1619.

12. Arras G, Usai M. Fungitoxic activity of essential oils against four postharvest citrus pathogens: chemical analysis of Thymus capitatus oil and its effect in sub-atmospheric pressure conditions. J Food Prot. 2001;64: $1025-1029$.

13. Panizzi L, Flamini G, Cioni PL, Morelli I. Composition and antimicrobial properties of essential oil of four Mediterranean lamiaceae. J Ethnopharmacol. 1993;39:167-170.

14. Kalemba D, Kunicka A. Antibacterial and antifungal properties of essential oils. Curr Med Chem. 2003;10:813-829.

15. Debjit B, Sampath Kumar KP, Akhilesh Y, Shweta S, Shravan P, Amit Sankar D. Recent trends in Indian traditional herbs Syzygium aromaticum and its health benefits. J Pharmacogn Phytochem. 2012;1: $13-22$.

16. Nelson L, Rhodus DMD, Janna BA. An evaluation of a chemical cautery agent and an anti-inflammatory ointment for the treatment of recurrent aphthous stomatitis: a pilot study. Quintessence Int. 1996;29(12): 769-773.

17. Kutscher A, Zegarelli EV, Ronald N, Chilton NW, Mercadante JL. A new, long-lasting vehicle for the application of drugs to mucous membranes. J Am Dent Assoc. 1961;62:666-669.

18. Reynolds JEF, Prasad AB. Orabase. In: Reynolds JEF, Prasad AB, editors. Martindale, the Extra Pharmacopoeia. London: The Pharmaceutical Press; 1982:951.

19. Mutimer M, Riffkin C, Hill JA, Cyr GN. Modern ointment base technology. I. Properties of hydrocarbon gels. J Am Pharm Assoc. 1956;45(2 pt 1): 101-105.

20. Hanane EH, Nadya L, Katim A, et al. Antioxidant properties and total phenolic content of three varieties of carob tree leaves from Morocco. Rec Nat Prod. 2010;4(4):193-204.

21. Aml M, Fathy M, El-Shanawany S. Formulation and in vitro evaluation of fluconazole topical gels. Br J Pharm Res. 2013;3(3):293-313.
22. Bhardwaj R, Blanchard J. Controlled-release delivery system for the a-MSH analog melanotan-I using poloxamer 407. J Pharm Sci. 1996; 85:915-919.

23. Zhang L, Parsons DL, Navarre C, Kompella UB. Development and in-vitro evaluation of sustained release poloxamer 407 (P407) gel formulations of ceftiofur. J Control Release. 2002;85(1-3):73-81.

24. Wang HF, Wang YK, Yih KH. DPPH free-radical scavenging activity, total phenolic contents and chemical composition analysis of forty-two kinds of essential oils. J Food Drug Anal. 2009;17(5):386-395.

25. Chary RB, Vani G, Rao YM. In vitro and in vivo adhesion testing of mucoadhesive drug delivery systems. Drug Dev Ind Pharm. 1999;25(5): 685-690.

26. Varshosaz J, Tavakoli N, Saidian S. Development and physical characterization of a periodontal bioadhesive gel of metronidazole. Drug Deliv. 2002;9(2):127-133.

27. Nakamura F. In vitro and in vivo nasal mucoadhesion of some water soluble polymers. Int J Pharm. 1996;134:173-181.

28. Attia MA, El-Gibaly I, Shaltout SE, Fetih GN. Transbuccal permeation, anti-inflammatory activity and clinical efficacy of piroxicam formulated in different gels. Int J Pharm. 2004;276:11-28.

29. El-Leithy ES, Shaker DS, Ghorab MK, Abdel-Rashid RS. Evaluation of mucoadhesive hydrogels loaded with diclofenac sodium-chitosan microspheres for rectal administration. AAPS Pharm Sci Tech. 2010; 11(4):1695-1702.

30. Marwa H, Ghada FM. Evaluation of topical gel bases formulated with various essential oils for antibacterial activity against methicillin-resistant Staphylococcus aureus. Trop J Pharm Res. 2013;12(6):877-884.

31. Pinto E, Pina-Vaz C, Salgueiro L, et al. Antifungal activity of the essential oil of Thymus pulegioides on Candida, Aspergillus and dermatophyte species. J Med Microbiol. 2006;55:1367-1373.

32. Cortés-Rojas DF, de Souza CR, Oliveira WP. Clove (Syzygium aromaticum): a precious spice. Asian Pac J Trop Biomed. 2014;4(2):90-96.

33. Ivan A, David VB, Roumiana PS, et al. Extraction of Thymol from different varieties of thyme plants using green solvents. In: III Iberoamerican Conference on Supercritical Fluids Cartagena de Indias, Colombia; 2013.

34. Mohd S, Birhanu D, Tanweer A. Antimicrobial activity of essential oils extracted from medicinal plants against the pathogenic microorganisms: a review. Issues Biol Sci Pharm Res. 2014;2(1):001-007.

35. Pokharkar VB, Shekhawat PB, Dhapte VV, Mandpe LP. Development and optimization of eugenol loaded nanostructured lipidcarriers for periodontal delivery. Int J Pharm Pharm Sci. 2011;3(4):138-143.

36. Pinto E, Vale-Silva L, Cavaleiro C, Salgueiro L. Antifungal activity of the clove essential oil from Syzygium aromaticum on Candida, Aspergillus and dermatophyte species. J Med Microbiol. 2009;58:1454-1462.

37. Nirav R. To study the herbalism of thyme leaves. Int J Pharm Ind Res. 2012;2(3):252-258.

38. Yajaman S, Ketousetuo K, Bandyopadhyay AK. Buccal bioadhesive drug delivery - a promising option for orally less efficient drugs. J Control Release. 2006;114:15-40.

39. Pia Gabre BE, Dowen B, Lars G. Oral retention of flouride from a mucosa adhesive paste (Orabase ${ }^{\mathrm{TM}}$ ) supplemented with $\mathrm{NaF}$ - a pilot study. Oral Health Prev Dent. 2005;3(3):159-163.

40. Sarunyoo S, Naranut R, Nipapat C. In vitro studies of mucoadhesiveness and release of nicotinamide oral gels prepared from bioadhesive polymers. World Acad Sci Eng Technol. 2009;31:113-120.

41. Fatma AI. Design and in vitro evaluation of polymeric formulae of simvastatin for local bone induction. Drug Dev Ind Pharm. 2006;32: 1199-1206.

42. EL Laithy H, EL Shaboury KMF. The development of cutina lipogels and gel microemulsion for topical administration of fluconazole. AAPS Pharm Sci Tech. 2002;3:1-9.

43. Mahrous G. Formulation and evaluation of fluconazole and ketorolac buccoadhesive dosage forms, Thesis in Pharmaceutics, Faculty of Pharmacy, Assuit, Egypt, 2006.

44. Usra ISA. Spectrophotometric determination of thymol in pharmaceuticals with Gibb's reagent. J Edu Sci. 2009;22(4):17-26. 
45. Schäfer K, Mehnertb W, Korting HC. Lipid nanoparticles for improved topical application of drugs for skin diseases. Adv Drug Deliv Rev. 2007; 59:427-443

46. Yuan H, Wang LL, Du YZ, You J, Hu FQ, Zeng S. Preparation and characteristics of nanostructured lipid carriers for controlreleasing progesterone by melt- emulsification colloids and surfaces B. Biointerfaces. 2007;60:174-179.

47. Nayak BS, Nayak UK, Patro KB. Bioadhesive vaginal gel for prolong release of metronidazole: formulation development, characterization and comparative study with marketed formulation. Int J Pharm Sci Technol. 2009;2(1):30-36.
48. Bansal K, Rawat MK, Jain A, Rajput A, Chaturvedi TP, Singh S. Development of satranidazole mucoadhesive gel for the treatment of periodontitis. AAPS Pharm Sci Tech. 2009;10(3):716-723.

49. Mogahed AM. Evaluation of topical application of thyme and clove essential oils in treatment of acute atrophic candidiasis, Thesis in oral medicine, periodontology, oral diagnosis and radiology department, faculty of dentistry, Alexandria University, Alexandria, 2012.

\section{Publish your work in this journal}

Drug Design, Development and Therapy is an international, peerreviewed open-access journal that spans the spectrum of drug design and development through to clinical applications. Clinical outcomes, patient safety, and programs for the development and effective, safe, and sustained use of medicines are a feature of the journal, which has also been accepted for indexing on PubMed Central. The manuscript management system is completely online and includes a very quick and fair peer-review system, which is all easy to use. Visit http://www.dovepress.com/testimonials.php to read real quotes from published authors.

Submit your manuscript here: http://www.dovepress.com/drug-design-development-and-therapy-journal 